Urologe $2022 \cdot 61: 167-172$

https://doi.org/10.1007/s00120-021-01619-0

Angenommen: 13. Juli 2021

Online publiziert: 23. August 2021

(c) Der/die Autor(en) 2021

\section{Radikale pelvine Tumorchirurgie bei Patienten mit einem lokal fortgeschrittenen, sympto- matischen kastrationsresistenten Prostatakarzinom}

\author{
Saskia Kanzelmeyer ${ }^{1}$. Mark Bludau ${ }^{3}$. David Johannes Karl Paul Pfister ${ }^{1}$. \\ Axel Heidenreich ${ }^{1,2}$ \\ ${ }^{1}$ Klinik und Poliklinik für Urologie, Uro-Onkologie, spezielle urologische und roboter-assistierte Chirurgie, \\ Universitätsklinikum Köln, Köln, Deutschland \\ ${ }^{2}$ Klinik für Urologie, Medizinische Universität Wien, Wien, Österreich \\ ${ }^{3}$ Klinik und Poliklinik für Allgemein-, Viszeral-, Tumor- und Transplantationschirurgie, Universitätsklinikum \\ Köln, Köln, Deutschland
}

Zusammenfassung

Ziel der Arbeit: Die retrospektive Evaluation der klinischen Ergebnisse nach palliativer pelviner Tumorchirurgie (ppTC) bei subvesikalen und supravesikalen Komplikationen eines lokal symptomatisch fortgeschrittenen kastrationsresistenten (CRPC) Prostatakarzinoms.

Patienten und Methoden: Insgesamt 84 Patienten mit lokal fortgeschrittenem und symptomatischem CRPC erhielten eine radikale Zystoprostatektomie $(n=71$, $83,3 \%$ ) oder anteriore und posteriore Exenteration ( $n=13,16,7 \%)$. Das lokale Staging erfolgte mittels MRT des Beckens, Zystoskopie und Rektoskopie. Ein systemisches Staging erfolgte mittels Computertomographie von Thorax, Abdomen, Becken sowie Skelettszintigraphie. Die perioperativen Komplikationen wurden nach Clavien-DindoKlassifikation evaluiert. Das primäre Studienziel war das symptomfreie Überleben (sÜL, Fehlen von Symptomen am unteren oder oberen Harntrakt, fehlende endoluminale oder perkutane Intervention).

Ergebnisse: Nach einem medianen Follow-up von 43,5 (3-139) Monaten betrug das sÜL nach einem und drei Jahren 95,2\% bzw. 86,7\%. Insgesamt 86,7\% der Patienten blieben für ihre gesamte verbleibende Lebensdauer bezüglich lokaler Symptome beschwerdefrei. Das OS nach einem und drei Jahren betrug 92,9\% bzw. 54,7\%. ClavienDindo-Grad-2-, -3- und -4-Komplikationen ergaben sich in 19 (22,6\%), 7 (8,3\%) bzw. 3 $(3,6 \%)$ Patienten.

Schlussfolgerungen: Die ppTC ist mit einer geringen Komplikationsrate möglich und führt zu deutlicher Symptomlinderung bei ca. $90 \%$ der Patienten, von denen $>80 \%$ für die verbleibende Lebenszeit beschwerdefrei verbleiben. Voraussetzung sind die Patientenauswahl, ein interdisziplinäres Vorgehen und eine entsprechende chirurgische Expertise.

\title{
Schlüsselwörter
}

Radikale Prostatektomie · Radikale Zystoprostatektomie · Vordere Exenteration · Hintere Exenteration Palliative Chirurgie 
Tab. 1 Charakteristika der 84 Patienten nach palliativer, radikaler pelviner Tumorchirurgie (ppTC)

\begin{tabular}{|c|c|}
\hline Parameter & $\begin{array}{l}\text { Patientenkollektiv } \\
(n=83)\end{array}$ \\
\hline Mittleres Alter (Jahre) & $69,2(56-82)$ \\
\hline \multicolumn{2}{|l|}{ Primärtherapie } \\
\hline $\begin{array}{l}\text { Radikale Prostatekto- } \\
\text { mie }\end{array}$ & $8(9,5 \%)$ \\
\hline $\begin{array}{l}\text { Perkutane Radiothera- } \\
\text { pie (72-81 Gy) }\end{array}$ & $46(54,8 \%)$ \\
\hline Androgendeprivation & $30(35,7 \%)$ \\
\hline \multicolumn{2}{|l|}{ Sekundärtherapie } \\
\hline Salvage RT + ADT + API & $34(40,5 \%)$ \\
\hline ADT + API+ Docetaxel & $30(35,7 \%)$ \\
\hline $\begin{array}{l}\text { ADT + API + Zweitlinie } \\
\text { API }\end{array}$ & $20(23,8 \%)$ \\
\hline \multicolumn{2}{|c|}{ ECOG-Performance-Status } \\
\hline 0 & $66(78,6 \%)$ \\
\hline 1 & $18(21,4 \%)$ \\
\hline \multicolumn{2}{|l|}{ Metastasenlokalisation $^{a}$} \\
\hline $\begin{array}{l}\text { Lymphknoten- } \\
\text { metastasen }\end{array}$ & $26(30,9 \%)$ \\
\hline Viszerale Metastase & $7(8,3 \%)$ \\
\hline Ossäre Metastasen & $59(70,2 \%)$ \\
\hline $\mathrm{nmCRPC}$ & $12(14,3 \%)$ \\
\hline \multicolumn{2}{|l|}{ Laborbefunde } \\
\hline PSA (ng/ml) & $31,4(9,8-157,3)$ \\
\hline Testosteron (ng/dl) & $29,4(17,1-41,4)$ \\
\hline Hämoglobin (g/dl) & $11,3(8,8-12,3)$ \\
\hline $\mathrm{LDH}(\mathrm{U} / \mathrm{I})$ & $\begin{array}{l}182,3 \\
(89,1-324,5)\end{array}$ \\
\hline $\mathrm{CRP}(\mathrm{mg} / \mathrm{l})$ & $6,3(<5,0-67,2)$ \\
\hline \multicolumn{2}{|c|}{$\begin{array}{l}\text { RT Strahlentherapie, } A D T \text { Androgendepri- } \\
\text { vation, } A R I \text { Andrognezeptorinhinbitor, } \mathrm{nm} \text { - } \\
C R P C \text { nichtmetastasiertes kastrationsresisten- } \\
\text { tes Prostatakarzinom, } L D H \text { Lactdehydrogen- } \\
\text { ase, } C R P \text { C-reakitves Protein } \\
{ }^{2} \text { Mehrfachlokalisationen sind möglich }\end{array}$} \\
\hline
\end{tabular}

\section{Einleitung}

Das Prostatakarzinom (PCA) kann bei lokal fortgeschrittenem, aggressivem Tumorwachstum nach primärer Lokaltherapie trotz initiierter systemischer medikamentöser Tumortherapie konsekutiv die Harnblase und/oder das Rektum infiltrieren [1]. Eine Infiltration dieser Organe geht häufig mit schwerwiegenden Symptomen wie rektalen Blutungen, Makrohämaturie \pm Blasentamponade, Harnverhalt, perinealen Schmerzen oder Hydronephrose einher.

Die überwiegende Mehrzahl dieser Patienten wird konservativ symptomatisch mittels palliativer TURP, transurethraler oder suprapubischer Katheteranlage bzw. endoluminaler Harnleiterschienung oder perkutaner Nephrostomie behandelt. Entsprechend aktueller retrospektiver Untersuchungen gehen alle diese Maßnahmen trotz vorübergehender Symptomenreduktion mit einer Einschränkung der Lebensqualität einher [2]. Eine effektive Kontrolle der lokalen Symptomatik wirkt sich zwar nicht auf das Gesamtüberleben aus, geht aber mit einer signifikanten Verbesserung der Lebensqualität einher und ist somit als Therapieziel definiert [3, 4].

Die palliative, radikale pelvine Tumorchirurgie (ppTC) stellt eine interdisziplinäre Behandlungsmöglichkeit dar, die bei adäquat ausgewählten Patienten mit lokal fortgeschrittenem und symptomatischem kastrationsresistenten Prostatakarzinom (CRPC) mit einer langfristigen Symptomkontrolle einhergehen kann.

Wir berichten über ein Kollektiv von 84 Patienten, bei denen eine ppTC unterschiedlicher Ausdehnung durchgeführt wurde und wir analysierten das symptomfreie Überleben, das Gesamtüberleben sowie die perioperative Komplikationsrate.

\section{Patienten und Methoden}

Es erfolgte eine retrospektive Analyse von 84 Patienten, die zwischen 2008 und 2021 bei lokal fortgeschrittenem, symptomatischem CRPC mittels ppTC an den jeweiligen Institutionen der Operateure behandelt wurden.

Die Indikation zur ppTC wurde bei symptomatischer Infiltration von Blase und/ oder Rektum unter laufender systemischer Therapie bei Vorliegen signifikanter Symptome wie rezidivierende Harnverhaltungen, Makrohämaturien mit/ohne Blasentamponade oder Bluttransfusionen, schmerzhafte Beckenbodeninfiltration, rektaler Obstruktion mit signifikanten Defäkationsproblemen oder obstruktivem Ileus gestellt.

Einschlusskriterien für den operativen Eingriff waren: ECOG-Performance-Status von 0-1, ASA-Status $\leq 2$, Lebenserwartung $>1$ Jahr und Symptome der lokalen Progression (rezidivierende Makrohämaturien mit oder ohne Bluttransfusion, resistente Beckenschmerzsymptomatik, obstruktiver
Ileus, rezidivierende Harnverhaltung, signifikante Obstruktion des oberen oder unteren Harntraktes) trotz fortlaufender systemischer medikamentöser Tumortherapie ohne Nachweis einer systemischen Progression.

Präoperativ wurden die folgenden Laborparameter evaluiert: PSA, Albumin, alkalische Phosphatase, Leber- und Nierenfunktionsparameter, C-reaktives Protein (CRP), Hämoglobin $(\mathrm{Hb})$, Leukozyten, Thrombozyten und Laktatdehydrogenase (LDH). Der Testosteronwert musste im Kastrationsniveau $<50 \mathrm{ng} / \mathrm{dl}$ gelegen sein.

Die Patienten erhielten zur Beurteilung der lokalen und systemischen Ausdehnung des CRPC eine umfassende Bildgebung mit Computertomographie von Thorax, Abdomen und Becken, Kernspintomographie der Prostata und des Beckens sowie Skelettszintigraphie. Eine ${ }^{68} \mathrm{Ga}$-PSMA-PET/CT-Untersuchung wurde aufgrund der fehlenden therapeutischen Konsequenzen nur bei 17/84 Patienten durchgeführt.

Im Falle Defäkationsbeschwerden erhielten alle Patienten eine Rektoskopie zum Ausschluss einer rektalen Infiltration.

Die radikale Zystoprostatektomie mit inkontinenter Harnableitung stellte Therapie der Wahl bei Infiltration der Harnblase dar (•Tab. 1). Im Falle einer rektalen Tumorinfiltration erfolgte eine anteriore und posteriore Exenteration als En-bloc-Resektion mit inkontinenter Harnableitung und einer endständigen Kolostomie.

Positive Schnittränder wurden durch den Nachweis von PCA-Zellen in der markierten Oberfläche von Prostata, Blase und/ oder Rektum definiert.

Alle Patienten erhielten peri- und postoperativ eine Antithromboseprophylaxe (Enoxaparin $40 \mathrm{mg} / \mathrm{Tag}, 21$ Tage) und Antibiose mit Metronidazol (400 mg 2-mal täglich, 3 Tage) und Cefuroxim (500 mg 3-mal täglich, 7 Tage).

Gemäß unseres klinikinternen Standards nach Anlage eines IC wurden die eingelegten Ureterkatheter für 7 Tage belassen und erst bei Nachweis einer patenten ureteroilealen Anastomose via retrograder Darstellung entfernt.

Die perioperativen Komplikationen wurden mittels Clavien-Dindo klassifiziert [5]. 
Tab. 2 Peri-und postoperative Ergebnisse nach palliativer pelviner Tumorchirurgie (ppTC)

\begin{tabular}{|c|c|}
\hline Variablen & $n(\%)$ \\
\hline \multicolumn{2}{|l|}{ Art der ppTC } \\
\hline Anteriore Exenteration & $71(83,3)$ \\
\hline Totale Exenteration & $13(16,7)$ \\
\hline \multicolumn{2}{|l|}{ Art der Harnableitung } \\
\hline Ileumconduit & $78(92,8)$ \\
\hline Ureterokutaneostomie & $6(7,2)$ \\
\hline \multicolumn{2}{|c|}{ Pathohistologisches Staging bei ppTC } \\
\hline pT3 & $10(11,9)$ \\
\hline pT4 & $74(88,1)$ \\
\hline pNO & $2(2,4)$ \\
\hline $\mathrm{pN}+$ & $21(25)$ \\
\hline $\mathrm{pNx}$ & $61(72,6)$ \\
\hline R1 & $37(44,1)$ \\
\hline Mittlere Operationszeit & $\begin{array}{l}271 \\
(210-310) \\
\text { Minuten }\end{array}$ \\
\hline \multicolumn{2}{|l|}{ Komplikationen } \\
\hline Clavien-Grad 2 & $19(22,6)$ \\
\hline Clavien-Grad 3 & $7(8,3)$ \\
\hline Wundrevision & $3(3,6)$ \\
\hline $\begin{array}{l}\text { Ureteroileale Anastomosen- } \\
\text { insuffizienz, DJ-Stent }\end{array}$ & $2(2,4)$ \\
\hline Fasziendehiszenz & $2(2,4)$ \\
\hline Clavien-Grad 4 & $3(3,6)$ \\
\hline Lungenarterienembolie & $1(1,2)$ \\
\hline Obstruktionsileus & $1(1,2)$ \\
\hline Darmperforation & $1(1,2)$ \\
\hline
\end{tabular}

Bei allen Patienten wurde die systemische medikamentöse Tumortherapie kontinuierlich fortgesetzt. Die tumorspezifische Nachsorge der Patienten umfasste typischerweise in 3-monatlichen Intervallen: PSA-Wertkontrolle, körperliche Untersuchung und Sonographie des oberen Harntrakts. Bei signifikanter PSAProgression wurde zur Ausbreitungsdiagnostik eine Bildgebung mittels CT Thorax, Abdomen, Becken sowie einer Skelettszintigraphie sowie ab 2018 in Einzelfällen mittels ${ }^{68} \mathrm{Ga}$-PSMA-PET/CT veranlasst.

Das symptomfreie Überleben (sÜL) wurde definiert als ein Fehlen von Symptomen am oberen oder unteren Harntrakt bzw. das Fehlen von endoluminalen oder perkutanen Interventionen.

\section{Statistik}

Die statistische Auswertung erfolgte STATA 16 (Stata Corp., College Station, TX, USA).

Die Ergebnisse wurden als signifikant gewertet, wenn sich im zweiseitigen Test ein $p$-Wert $<0,05$ errechnete. KaplanMeier-Analysen wurden verwendet, um das symptomfreie Überleben und das Gesamtüberleben zu evaluieren. Univariate und multivariate Cox-Regressionsanalysen wurden durchgeführt, um eine Assoziation zwischen ECOG-Performance-Status sowie präoperativen Laborparametern mit den perioperativen Ergebnissen zu evaluieren.

\section{Ergebnisse}

Die Patientencharakteristika sind in $\bullet$ Tab. 1 dargestellt. 8 bzw. 46 Patienten waren einer radikalen Prostatektomie bzw. einer perkutanen Strahlentherapie als primärer lokaler Therapie zugeführt worden, während 30 Patienten eine primäre ADT ohne lokale Therapie erhielten.

Alle Patienten wiesen ein CRPC auf (Serumtestosteronkonzentration $<50 \mathrm{ng} / \mathrm{dl}$, konsekutiv ansteigende PSA-Werte, Progression in der bildgebenden Diagnostik). Folgende therapierefraktäre Symptome führten zur ppTC: subvesikale Obstruktion in $69(82,1 \%)$, supravesikale Obstruktion in $46(54,8 \%)$ bzw. eine Kombination aus beidem, rezidivierende Makrohämaturien mit Blasentamponaden und/oder Transfusionspflichtigkeit in 29 (34,5\%) bzw. ein obstruktiver Subileus/lleus in 8 (9,5\%).

Das Ausmaß der lokalen Tumorausdehnung variierte zwischen einer Infiltration des Blasenhalses über die Infiltration der dorsalen Blasenwand bis hin zur Infiltration von Blase und Rektum (-Abb. 1 und 2), sodass bei 71 bzw. 13 Patienten eine radikale Zystoprostatektomie mit Harnableitung bzw. eine totale Exenteration mit Harnableitung und Anlage eines Kolostomas erfolgte. Pathohistologisch zeigte sich bei allen Patienten ein Adenokarzinom der Prostata und in keinem Falle lag ein reines oder dominierendes neuroendokrines Prostatakarzinom vor.

Zum Zeitpunkt der ppTC wiesen 7 (8,3\%) Patienten viszerale Metastasen auf, 65 (77,4\%) Patienten hatten retrope- ritoneale und/oder ossäre Metastasen und bei 12 (14,3\%) lag ein metastasenegatives, symptomatisches lokales Rezidiv mit einer PSA-Verdopplungszeit > 12 Monaten vor.

In - Tab. 2 sind die perioperativen onkologischen und funktionellen Ergebnisse nach ppTC dargestellt. $83 \%$ der Patienten wurden mittels radikaler Zystoprostatektomie und Anlage einer inkontinenten Harnableitung therapiert; bei $17 \%$ der Patienten war eine anteriore und posteriore Exenteration erforderlich. Es traten keine Clavien-Dindo-Grad-5-Komplikationen auf. Clavien-Grad-2-, -3- und -4-Komplikationen entwickelten sich in $22,6 \%, 8,3 \%$ bzw. 3,6\% während der ersten 90 postoperativen Tage (- Tab. 2). Der mittlere Hospitalisationsdauer betrug 20,7 (9-34) Tage. 6 Patienten (5,8\%) entwickelten perioperativ eine rapide Tumorprogression und verstarben innerhalb von 6 Monaten postoperativ. Alle diese Patienten hatten hohe CRP-Werte (Mittelwert 53,4 \pm 12,3 mg/dl) und hohe PSA-Serumwerte (Mittelwert $95,6 \pm 19,3 \mathrm{ng} / \mathrm{ml}$ ).

Bei einer medianen Nachbeobachtungszeit von 43,5 (3-139) Monaten verblieben 75 (89,2\%) Patienten symptomfrei. Das 1- und 3-Jahres-SFS betrug 95,2\% bzw. 89,2\% (- Abb. 3). 6 Patienten $(5,8 \%)$ benötigten aufgrund einer Obstruktion der oberen Harnwege durch progrediente retroperitoneale Lymphadenopathie $(n=4)$ oder Stenose der ureteroilealen Anastomose $(n=2)$ eine endoluminale Harnleiterschienung. Ein Patient entwickelte während der Nachsorge einen obstruktiven Ileus aufgrund einer Peritonealkarzinose und 2 weitere Patienten entwickelten einen obstruktiven lleus aufgrund von Dünndarmadhäsionen. 38 (45,2\%) Patienten verstarben während der Nachbeobachtungsphase. Die Überlebensrate nach 1 Jahr sowie 3 Jahren betrug $92,9 \%$ bzw. 54,7\%. Von den 46 Patienten waren nach 3 Jahren noch immer 42 $(86,7 \%)$ im Urogenitaltrakt symptomfrei.

\section{Diskussion}

Trotz moderner lebensverlängernder und onkologisch effektiver Medikationen zur Behandlung des CRPC bleibt das lokal fortgeschrittene und symptomatische PCA eine therapeutische Herausforderung [6, 7]. Obwohl ADT mit oder ohne systemi- 

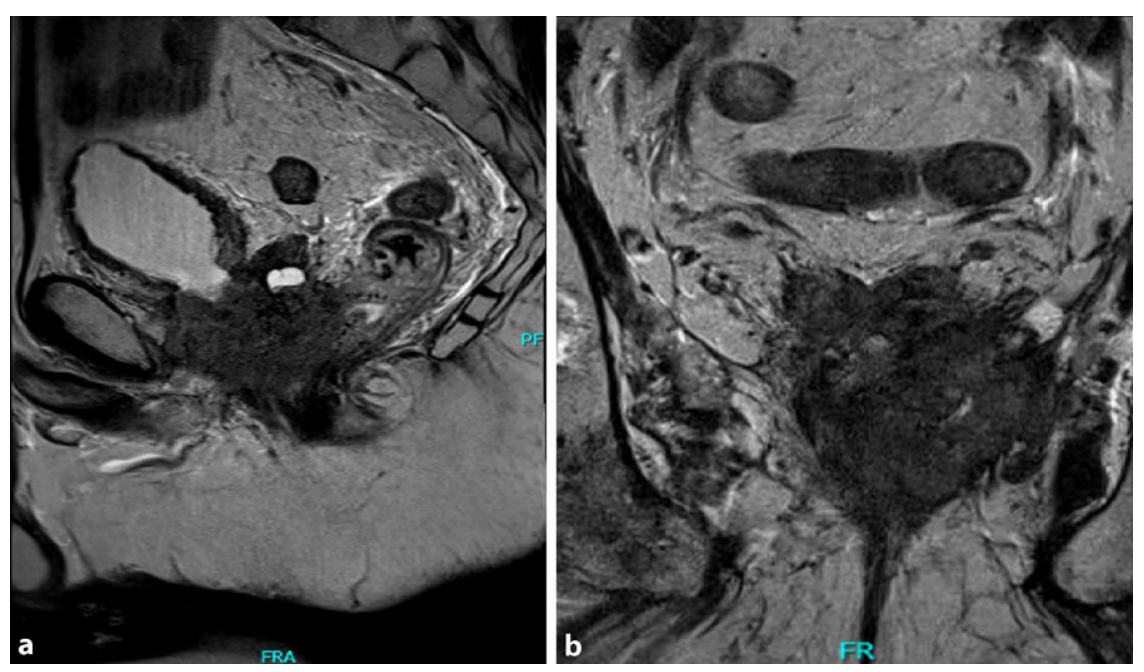

Abb. $1 \Delta$ Darstellung eines lokal fortgeschrittenen CRPC mit Infiltration der Harnblase und des Rektums in der coronaren (a) und der sagitalen Schocht (b)

scher Kombinationstherapien die Progression von Systemmetastasen verlangsamen und kontrollieren können, sind ist eine intrapelvine Progression mit signifikanter Symptomatik nicht selten. Patrikidou et al. berichten, dass etwa zwei Drittel der Männer mit neu diagnostiziertem metastasiertem PCA unter lokoregionären Symptomen leiden, die in $60 \%$ der Fälle chirurgische Interventionen wie die palliative TURP (36\%) oder endourologische Maßnahmen $(25 \%)$ erfordern [3]. Ähnliche Daten wurden von Won et al. angeführt [8], die eine Kohorte von 263 Patienten mit CRPC analysierten, von denen $45 \%$ eine signifikante subvesikale Obstruktion oder $\mathrm{Hy}$ dronephrose entwickelten, wenn der Primärtumor nicht operativ, sondern mit ADT oder ADT plus RT behandelt wurde. Diese Symptome haben zwar keinen Einfluss auf das Überleben, haben aber negative Auswirkungen auf die Lebensqualität. Aus et al. [9] zeigten, dass $61 \%$ der CRPC-Patienten mindestens einmal in ihrer verbleibenden Lebenszeit palliativ operiert werden müssen, wenn der Primärtumor initial konservativ behandelt wurde. Die Patienten wurden in ihrem letzten Lebensjahr im Mittel 5 Wochen stationär zur Behandlung einer durch lokale Tumorprogression verursachten Symptomatik hospitalisiert. Auch in neueren prospektiven klinischen Studien wurde bei einem Drittel der Patienten ein symptomatischer Lokalprogress trotz Kombinationstherapie aus ADT, anderen lebensverlängernden The- rapieoptionen und Radiatio der Prostata beobachtet [10]. Aufgrund der zunehmenden verbesserten medianen Überlebenszeiten von 4 bis 6 Jahren durch die modernen lebensverlängernden medikamentösen Therapiemaßnahmen ist zu erwarten, dass der Bedarf an chirurgischem Management von symptomatisch progredientem PCA zunehmen wird [11].

Die aktuelle Studie repräsentiert die größte Patientenkohorte, die sich einer ppTC bei symptomatischem, lokal progredientem CRPC unterzogen hat. Wir konnten zeigen, dass Patienten in gutem Allgemeinzustand (ECOG 0-1, ASA 2), mit einer Lebenserwartung $>1$ Jahr und ausschließlich lokalen Symptomen wenig signifikante operationsassoziierte Komplikationen erleiden und eine deutliche Symptomlinderung über mehr als $80 \%$ der verbleibenden Lebenszeit erfahren. Jedoch verstarben 6 Patienten in den ersten 6 perioperativen Monaten aufgrund einer raschen systemischen Tumorprogression, die möglicherweise auf eine paraneoplastische inflamatorische Aktivierung des Immunsystems zurückzuführen sein könnte. Zwei retrospektive Studien wiesen in diesem Zusammenhang darauf hin, dass erhöhte CRP-Werte und erniedrigte Albuminserumkonzentrationen mit einem schlechteren progressionsfreien und Gesamtüberleben bei metastasiertem hormonsensitiven bzw. kastrationsresistenten PCA assoziiert sind [12-14]. Erhöhte CRP-Werte weisen auf eine Akute-

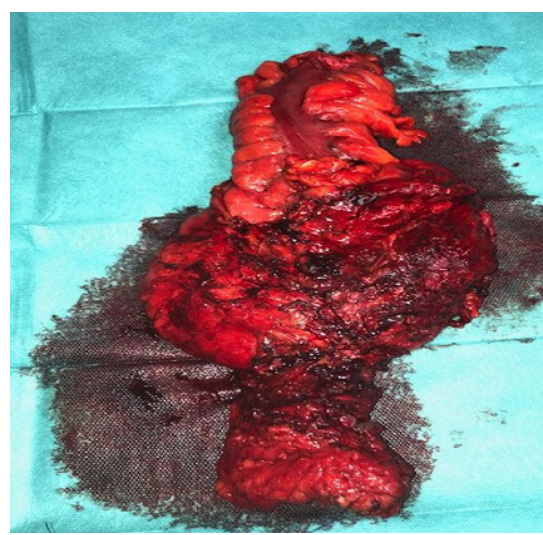

Abb. $2 \wedge$ Operationspräparat einer abdominoperinealen En-bloc-Resektion von Sigma, Harnblase, Prostata, Rektum und Anus

Phase-Reaktion hin, die durch die Freisetzung von IL-6 aus Prostatakrebszellen ausgelöst wird [13]. Auch wurde gezeigt, dass eine Erniedrigung des Albumins bzw. eine Erhöhung von CRP und IL-6 mit dem erhöhten Risiko eines kurzfristigen Todes innerhalb der ersten 6 postoperativen Monate nach einer größeren onkologischen Operation verbunden sind [14]. Diese Patienten profitieren eher nicht von einer Operation, sondern benötigen eine sekundäre systemische Therapie inklusive einer optimalen palliativen und supportiven Behandlung.

Die Rate an Grad-4-Komplikationen nach ppTC lag bei 3,6\% und ist vergleichbar mit perioperativen Komplikationsraten anderer radikaler tumorchirurgischer Eingriffe im Becken, sodass die ppTC ebenfalls mit geringer Morbidität zu realisieren ist $[15,16]$. Auch Grad-3-a-b-Komplikationen wurden nur in $8,3 \%$ der Fälle beobachtet, was sowohl der strengen präoperativen Selektion der Patienten, als auch der chirurgischen Erfahrung geschuldet ist. Dennoch muss über eine Gesamtrate von $20 \%$ signifikanter Komplikationen eine ausführliche präoperative Aufklärung erfolgen.

Bei der überwiegenden Mehrheit der Patienten konnte eine signifikante Reduktion der präoperativen Symptomatik für $>80 \%$ der verbleibenden Lebenszeit erreicht werden. Die ppTC schaffte in dieser Patientenkohorte einen lang anhaltenden Nutzen durch effizientes Tumor-Debulking. Die Lebensqualität konnte durch das Fehlen von wiederholten Wechseln von Harnleiterschienen oder perkutanen 


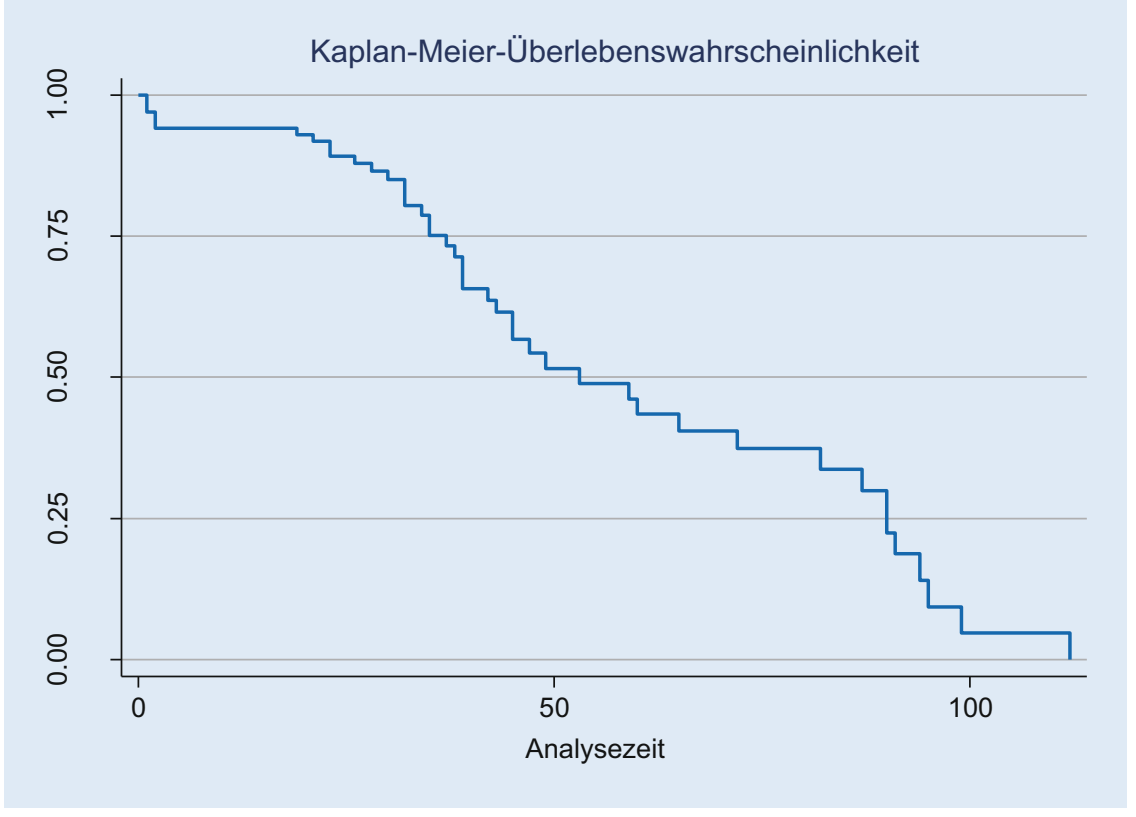

Abb. 3 ॥ Symptomfreies Überleben nach palliativer Beckenchirurgie

Nephrostomien deutlich verbessert werden $[17,18]$.

Unsere Ergebnisse stehen im Einklang mit früheren Studien, die gezeigt haben, dass weder ADT noch Chemotherapie eine adäquate Symptomlinderung bei der Behandlung des lokal fortgeschrittenen und symptomatischen CRPC erreichen können [19-21]. Leibovici et al. demonstrierten bei 30 von 38 Patienten $(88,2 \%)$ mit lokal fortgeschrittener Tumorerkrankung eine signifikante und dauerhafte Reduktion der Symptome nach palliativer Zystoprostatektomie [19]. Kamat et al. berichteten über 14 Patienten, die aufgrund einer rektalen Invasion durch ein PCA unter Miktionsbeschwerden und pelviner Schmerzsymptomatik litten und nach Exenteration alle beschwerdegelindert waren [20]. Unsere Daten zeigten kürzlich bei einer Kohorte von 38 Patienten eine langfristige Symptomlinderung bei $>80 \%$ der Patienten nach palliativer Zystektomie [21].

In allen Serien, einschließlich der unsrigen, variierten die Überlebensraten der Patienten nach ppTC zwischen unter 6 Monaten und mehreren Jahren. Dies verdeutlicht, dass die Patientenselektion von größter Bedeutung zu sein scheint, um Nutzen und Schaden der Operation abzuwägen und den palliativen Charakter der Maßnahme zu reflektieren.
Die palliative pelvine Strahlentherapie bei symptomatischem PCA kann als alternative Behandlungsoption zur ppTC bei Männern mit geringer lokoregionärer Tumorlast diskutiert werden [22, 23]. Allerdings wiesen alle von uns eingeschlossenen Patienten eine hohe Tumorlast auf, die durch eine RT nicht hätte kontrolliert werden können. Zwei Drittel der Patienten hatten sich bereits einer RT an der Prostata unterzogen.

Trotz der hohen Patientenzahlen ist unsere retrospektive Studie nicht frei von Limitationen. Die mittlere Nachbeobachtungszeit beträgt nur knapp 4 Jahre, wobei auch die begrenzte mittlere Gesamtüberlebenszeit bei Patienten mit CRPC berücksichtigt werden muss. Die positiven Ergebnisse könnten durch die oben beschriebene sorgfältige Patientenselektion verzerrt sein. Diese ist jedoch entscheidend, um signifikante behandlungsassoziierte Komplikationen, die die Lebensqualität beeinträchtigen oder sogar die Gesamtüberlebenszeit reduzieren könnten, zu vermeiden. Die fehlende konsequente Erfassung von Daten zur Lebensqualität mittels validierter Fragebögen stellt eine zusätzliche Limitation dar, da wir uns initial nur auf die Dokumentation der Symptomverbesserung fokussiert hatten.
Fazit für die Praxis

- Unsere Studie repräsentiert die größte Kohorte von Patienten, bei denen eine palliative pelvine Exenteration bei lokal fortgeschrittenem, symptomatischem kastrationsresistentem Prostatakarzinom (CRPC) durchgeführt wurde. Sie ist daher von besonderer Bedeutung und liefert klinisch wichtige Informationen für das Management dieser Patienten.

- Die präoperative Patientenselektion ist entscheidend, nur Patienten in gutem Allgemeinzustand, mit Low-volume-Metastasierung und normalen Albumin- und CRP-Serumkonzentrationen sollten hinsichtlich dieser Art der Operation beraten werden. Diese genannten Parameter sind jedoch streng zu beachten, sodass nur ein sehr ausgewähltes Patientenkollektiv für die ausgedehnte Resektion in Frage kommt.

- Der Nutzen ist hoch, mit einem symptomfreien Überleben $>80 \%$ der verbleibenden Lebenszeit. Unserer Erfahrung nach sollte die palliative pelvine Chirurgie mit radikaler Zystoprostatektomie und/oder posteriorer Exenteration als therapeutische Möglichkeit zur Symptomlinderung bei lokal fortgeschrittenem und symptomatischem CRPC standardisiert werden.

\section{Korrespondenzadresse}

Univ.-Prof. Dr. med. Axel Heidenreich Klinik und Poliklinik für Urologie, UroOnkologie, spezielle urologische und roboterassistierte Chirurgie, Universitätsklinikum Köln Kerpener Str. 62, 50937 Köln, Deutschland axel.heidenreich@uk-koeln.de

Funding. Open Access funding enabled and organized by Projekt DEAL.

\section{Einhaltung ethischer Richtlinien}

Interessenkonflikt. S. Kanzelmeyer, M. Bludau, D.J.K.P. Pfister und A. Heidenreich geben an, dass kein Interessenkonflikt besteht.

Für diesen Beitrag wurden von den Autoren keine Studien an Menschen oder Tieren durchgeführt. Für die aufgeführten Studien gelten die jeweils dort angegebenen ethischen Richtlinien.

Open Access. Dieser Artikel wird unter der Creative Commons Namensnennung 4.0 International Lizenz veröffentlicht, welche die Nutzung, Vervielfältigung, Bearbeitung, Verbreitung und Wiedergabe in jeglichem Medium und Format erlaubt, sofern Sie den/die ursprünglichen Autor(en) und die Quelle ordnungsgemäß nennen, einen Link zur Creative Commons Lizenz beifügen und angeben, ob Änderungen vorgenommen wurden. 
Die in diesem Artikel enthaltenen Bilder und sonstiges Drittmaterial unterliegen ebenfalls der genannten Creative Commons Lizenz, sofern sich aus der Abbildungslegende nichts anderes ergibt. Sofern das betreffende Material nicht unter der genannten Creative Commons Lizenz steht und die betreffende Handlung nicht nach gesetzlichen Vorschriften erlaubt ist, ist für die oben aufgeführten Weiterverwendungen des $\mathrm{Ma}$ terials die Einwilligung des jeweiligen Rechteinhabers einzuholen.

Weitere Details zur Lizenz entnehmen Sie bitte der Lizenzinformation auf http://creativecommons.org/ licenses/by/4.0/deed.de.

\section{Literatur}

1. Sweeney CJ, Chen YH, Carducci M et al (2015) Chemohormonal therapy in metastatic hormone-sensitive prostate cancer. $\mathrm{N}$ Engl J Med 373:737-746

2. Heidenreich A, Porres D, Pfister D (2015) The role of palliative surgery in castration-resistant prostate cancer. Oncol Res Treat 38:670-677

3. Patrikidou A, Brureau L, Casenave J et al (2015) Locoregional symptoms in patients with de novo metastatic prostate cancer: Morbidity, management, and disease outcome. Urol Oncol 33:202.e9-202.e17

4. Ferenschild FTJ, Vermaas M, Verhoef C et al (2009) Total pelvic exenteration for primary and recurrent malignancies. World J Surg 33:1502-1509

5. Dindo D, Demartines N, Clavien P (2004) Classification of surgical complications: a new proposal with evaluation in a cohort of 6336 patients and results of a survey. Ann Surg 240:205-213

6. Cornford P, Bellmunt J, Bolla M et al (2017) EAUESTRO-SIOG guidelines on prostate cancer. Part II: treatment of relapsing, metastatic, and castrationresistant prostate cancer. Eur Urol 71:630-642

7. Feyerabend S, Saad F, Li T et al (2018) Survival benefit, disease progression and quality-of-life outcomes of abiraterone acetate plus prednisone versus docetaxel in metastatic hormone-sensitive prostate cancer: a network meta-analysis. Eur J Cancer 103:78-87

8. Won ACM, Gurney H, Marx G et al (2013) Primary treatment of the prostate improves local palliation in men who ultimately develop castrate-resistant prostate cancer. BJU Int 112:E250-E255

9. Aus G, Hugosson J, Norlén L (1995) Need for hospital care and palliative treatment for prostate cancer treated with noncurative intent. J Urol 154:466-469

10. Parker CC, James ND, Brawley CD et al (2018) Systemic Therapy for Advanced or Metastatic Prostate cancer: Evaluation of Drug Efficacy (STAMPEDE) investigators. Radiotherapy to the primary tumour for newly diagnosed, metastatic prostate cancer (STAMPEDE): a randomised controlled phase 3 trial. Lancet 392:2353-2366

11. Chi KN, Agarwal N, Bjartell A et al (2019) Apalutamide for metastatic, castration-sensitive prostate cancer. NEngl J Med 381:13-24

12. Rocha P, Morgan CJ, Templeton AJ et al (2014) Prognostic impact of C-reactive protein in metastatic prostate cancer: a systematic review and meta-analysis. Oncol Res Treat 37:772-776

13. Arthur R, Williams R, Garmo H et al (2018) Serum inflammatory markers in relation to prostate cancer severity and death in the Swedish AMORIS study. Int J Cancer 142:2254-2262

\section{Pelvic exenteration in patients with locally advanced, symptomatic castration-resistant prostate cancer}

Goal: The retrospective evaluation of clinical outcomes after palliative pelvic exenteration (PPE) in patients with subvesical and supravesical complications due to symptomatic locally advanced castration-resistant prostate cancer (CRPC).

Patients and methods: In all, 84 patients with locally advanced and symptomatic CRPC underwent radical cystoprostatectomy $(n=71,83.3 \%)$ or anterior and posterior exenteration ( $n=13,16.7 \%$ ). Local staging was done via pelvic MRI (magnetic resonance imaging), cystoscopy, and rectoscopy. Systemic staging was performed by computed tomography of the thorax, abdomen pelvis, and skeletal scintigraphy. Perioperative complications were evaluated using the Clavien-Dindo classification. The primary study objective was symptom-free survival defined as absence of lower or upper urinary tract symptoms and absence of endoluminal or percutaneous intervention.

Results: After a median follow-up of 43.5 (3-139) months, symptom-free survival at 1 and 3 years was $95.2 \%$ and $86.7 \%$, respectively. In all, $86.7 \%$ of patients remained symptom-free for their remaining lifetime with respect to local symptoms. Overall survival at 1 and 3 years was $92.9 \%$ and $54.7 \%$, respectively. Clavien-Dindo grade 2, 3, and 4 complications occurred in $19(22.6 \%), 7(8.3 \%)$, and $3(3.6 \%)$ patients, respectively. Conclusion: With adequate patient selection, PPE is possible with a low complication rate and results in significant symptom relief in the lower or upper genitourinary tract in about $90 \%$ of patients, of whom more than $80 \%$ remain symptom-free for the remainder of their lives. Prerequisites for favorable surgical outcomes are patient selection, an interdisciplinary approach, and appropriate surgical expertise.

\section{Keywords}

Radical prostatectomy - Radical cystoprostatectomy · Anterior pelvic exenteration · Posterior pelvic exenteration Palliative care

14. Nguyen DP, Li J, Tewari AK (2014) Inflammation and prostate cancer: the role of interleukin 6 (IL-6). BJU Int 113:986-992

15. Kumar A, Torres ML, Cliby WA et al (2017) Inflammatory and nutritional serum markers as predictors of peri-operative morbidity and survival in ovarian cancer. Anticancer Res 37:3673-3677

16. Mannas MP, Lee T, Forbes CM et al (2019) Predicting complications following radical cystectomy with the ACS NSQIP universal surgical risk calculator. World J Urol. https://doi.org/10.1007/s00345019-02915-3

17. Villari D, Nesi G, Della Melina A et al (2010) Radical retropubic prostatectomy for prostate cancer with microscopic bladder neck involvement: survival and prognostic implications. BJU Int 105:946-950

18. New F, Deverill S, Somani BK (2018) Role of percutaneous nephrostomy in end of life prostate cancer patients: a systematic review of the literature. Cent European JUrol 71:404-409

19. Bigum LH, Spielmann ME, Juhl G et al (2015) A qualitative study exploring male cancer patients' experiences with percutaneous nephrostomy. Scand JUrol 49:162-168

20. Leibovici D, Kamat AM, Pettaway CA et al (2005) Cystoprostatectomy for effective palliation of symptomatic bladder invasion by prostate cancer. JUrol 174:2186-2189

21. Kamat AM, Huang SF, Bermejo CE et al (2003) Total pelvic exenteration: effective palliation of perineal pain in patients with locally recurrent prostate cancer. JUrol 170:1868-1872

22. Heidenreich A (2016) Palliative radical (cysto) prostatectomy for locally advanced, symptomatic castration-resistant prostate cancer. Eur Urol Focus 2:478-479

23. Cameron MG, Kersten C, Guren MG et al (2014) Palliative pelvic radiotherapy of symptomatic incurable prostate cancer-a systematic review. Radiother Oncol 110:55-61 